\title{
Social top-down response modulation (STORM): a model of the control of mimicry in social interaction
}

\section{Yin Wang* and Antonia F. de C. Hamilton}

School of Psychology, University of Nottingham, Nottingham, UK

\author{
Edited by: \\ Chris Frith, Wellcome Trust Centre \\ for Neuroimaging at University \\ College London, UK \\ Reviewed by: \\ Marcel Brass, Ghent University, \\ Belgium \\ Jack Van Honk, Utrecht University, \\ Netherlands \\ Geoff Bird, Birkbeck College, UK \\ *Correspondence: \\ Yin Wang, School of Psychology, \\ University of Nottingham, NG7 2RD, \\ Nottingham, UK. \\ e-mail: mirrorneuronwang@ \\ gmail.com
}

As a distinct feature of human social interactions, spontaneous mimicry has been widely investigated in the past decade. Research suggests that mimicry is a subtle and flexible social behavior which plays an important role for communication and affiliation. However, fundamental questions like why and how people mimic still remain unclear. In this paper, we evaluate past theories of why people mimic and the brain systems that implement mimicry in social psychology and cognitive neuroscience. By reviewing recent behavioral and neuroimaging studies on the control of mimicry by social signals, we conclude that the subtlety and sophistication of mimicry in social contexts reflect a social top-down response modulation (STORM) which increases one's social advantage and this mechanism is most likely implemented by medial prefrontal cortex (mPFC). We suggest that this STORM account of mimicry is important for our understanding of social behavior and social cognition, and provides implications for future research in autism.

Keywords: mimicry, imitation, social cognition, social interaction, medial prefrontal cortex, mirror neurons, mentalising, top-down

\section{INTRODUCTION}

Human social interaction is complex and dynamic (Hari and Kujala, 2009). Individuals communicate with each other by means of multiple verbal and nonverbal behaviors, which rapidly change from moment to moment. Unraveling mechanisms underlying the subtlety of social behaviors is important for our understanding of the nature of human social interaction.

One remarkable nonverbal behavior during social interactions is spontaneous mimicry (van Baaren et al., 2009). People have a tendency to unconsciously imitate other's behaviors (Chartrand and van Baaren, 2009). In the past decade, this spontaneous mimicry has become the key focus of research in social psychology and cognitive neuroscience (Heyes, 2009), and has been regarded as a paradigm for exploring the complexity of human social interaction. Investigations of the causes, consequences and brain basis of mimicry have been widely carried out (Chartrand and van Baaren, 2009). For example, social psychology suggests that mimicry has positive consequences on social interaction; it increases liking and affiliation between interaction partners and makes communication more smooth and enjoyable (Chartrand and Bargh, 1999). Cognitive neuroscience further suggests that mimicry is based on the mirror neuron system (MNS) (Catmur et al., 2008, 2009; Heyes, 2011a). This system provides a direct link between perception and action where observing an action automatically activates the motor representation of that action (Brass and Heyes, 2005) and this link is most likely developed by associative sequence learning ("the ASL theory," Heyes, 2001, 2011a; Catmur et al., 2007, 2008, 2009).

However, two key questions still remain unclear. First, what is the purpose of mimicry? Although the ASL theory clearly elucidates how we develop the ability to mimic, it does not directly explain under what circumstances we will mimic and why we mimic to different degrees in different situations. Second, what brain mechanisms control and implement mimicry responses? In this article we aim to address these two questions by reviewing cutting-edge research on the control of mimicry by social signals. In the first part, we give a brief outline of past theories on the purpose of mimicry and emphasize that mimicry is a strategy for social advantage. We provide evidence that mimicry changes depending on the social context [i.e., social top-down response modulation (STORM)], and suggest that this subtle control may reflect a Machiavellian strategy for enhancing one's social standing. In the second part, we move to a neuroscience point of view and examine the information processing systems underlying the control of mimicry. We suggest that medial prefrontal cortex (mPFC) plays a key role in the control of mimicry in social contexts. Finally, we discuss the importance of the STORM model of mimicry in our understanding of social interaction and social cognition. We argue that subtly controlling when and who to mimic is essential to human competence in social interactions and suggest that impairment of this function could lead to social-communication disorders such as autism.

\section{WHY DO WE MIMIC? THEORIES OF MIMICRY}

Mimicry is a pervasive behavior in social interaction. People spontaneously copy a wide array of behavioral mannerisms from other individuals such as the postures, gestures, facial expressions, emotions, and languages (Chartrand and van Baaren, 2009). This "Chameleon effect" is not normally conscious controlled (Chartrand and Bargh, 1999) and most likely develops from longterm associative learning (Heyes, 2001, 2011a). Extensive research 
suggest that mimicry is a subtle and flexible behavior which is sensitive to social situations, the people involved, and the specific goals of the interaction at hand (Chartrand and van Baaren, 2009). The purpose (or function) of mimicry has long been debated by social psychologists and cognitive neuroscientists. Three major theories have been proposed so far.

First, the STORM theory of mimicry claims that mimicry is a strategic intervention to change the social world for selfadvancement (Lakin and Chartrand, 2003; Lakin et al., 2003). The theory assumes that if I mimic Anna, she unconsciously detects the mimicry and changes her attitude toward me. Thus, I can use mimicry as tool to make Anna like me, and will do this more if "Anna liking me" is to my social advantage. This implementation of mimicry is somewhat Machiavellian, in that it is strategic and driven by the anticipated social consequences of the action. The underlying mechanism could be unconscious and unintentional. Evidence in favor comes from studies of the positive consequences of mimicry, suggesting that being mimicked leads a participant to like the mimicker (Chartrand and Bargh, 1999), feel close to the mimicker (van Baaren et al., 2004), give more money to the mimicker (van Baaren et al., 2003a), and be more easily persuaded by the mimicker (Maddux et al., 2008).

The second theory claims that mimicry is a form of simulation, and functions to improve interactive alignment between two individuals and thus feed into a simulation theory of mind (Pickering and Garrod, 2004; Gallese, 2007, 2009; Niedenthal et al., 2010). For example, when I mimic Anna, that helps me understand how Anna feels and gives me better insight into her desires and intentions. Evidence in favor comes from studies showing that preventing automatic mimicry of facial expressions makes people slower to judge what expression is shown (Stel and van Knippenberg, 2008). Preventing mimicry also changes brain activation in response to seeing faces (Hennenlotter et al., 2009).

Finally, the third theory suggests that mimicry is largely an epiphenomena arising out of domain-general processes and has no specific social purposes or functions. This domain-general model is an extension of the ASL theory (Heyes, 2001) but is not explicitly stated or endorsed by Heyes. Under this model, mimicry is not necessary to be a special social behavior but could be implemented in just the same way as other over-learned non-social responses (Heyes, 2011b). Mimicry can be modulated by social signals, but it may assume that social modulation of mimicry is a side-effect of simple domain-general processes such as attention, conditioning, and disinhibition (Heyes, 2012a). Several sources of evidence suggest that mimicry is subject to general attentional effects (Chong et al., 2009; Heyes, 2011a, 2012b) and classical conditioning effects (Cook et al., 2012). This means that it is important to consider the null hypothesis that there is no dedicated and sophisticated mechanism for determining when and who to mimic.

Although all three theories acknowledge the essential role of associative learning in the development of mimicry, the positive contributions of mimicry to social interaction, and the flexibility and sensitivity of mimicry in social contexts (van Baaren et al., 2009; Press et al., 2011; Heyes, 2012a), they emphasize different functions of mimicry and thus have different predictions on empirical evidence. The simulation theory predicts that mimicry should be as fast and detailed as possible, to maximize interactive alignment (Pickering and Garrod, 2004). Thus, mimicry should be driven by the detailed contents of imitable features but not by social features that cannot be imitated (e.g., eye gaze and social group membership). In contrast, the STORM theory suggests more sophisticated mimicry, driven by integrative evaluation of all social features in the current interaction. If mimicry in the current situation can benefit one's social standing, individuals will increase mimicry; on the contrary, if mimicry becomes inappropriate at the present time, individuals will inhibit mimicry. The domain-general model suggests that mimicry is largely determined by prior sensorimotor experience and is strongly influenced by the properties of the stimulus (i.e., stimulus-driven; Heyes, 2012a,b). Modulation of mimicry by simple domaingeneral processes is possible, but the null hypothesis does not detail any sophisticated, consequence-driven control of mimicry. Here we review some recent data on mimicry from both social psychology and cognitive neuroscience, which leads us to support the STORM account.

\section{RESEARCH ON MIMICRY FROM SOCIAL PSYCHOLOGY}

Research in social psychology provides substantial evidence that mimicry is sophisticatedly directed by social signals (Chartrand and van Baaren, 2009) and this control of mimicry is for one's social advantage (Lakin et al., 2003). People increase mimicry toward those who are important for their social welfare. For example, participants show stronger mimicry to targets who are human but not robots (Longo and Bertenthal, 2009; Liepelt and Brass, 2010), who are attractive and nice (Likowski et al., 2008; van Leeuwen et al., 2009; Stel et al., 2010), who are powerful and have high social status (Cheng and Chartrand, 2003; Mastrop et al., in preparation), and who are friends and in-group members (Yabar et al., 2006; Bourgeois and Hess, 2008). People also increase mimicry when their social relationship is endangered. For example, participants show enhanced mimicry when they fail to affiliate with other individuals (Lakin and Chartrand, 2003) or when they are ostracized by their group members (Lakin et al., 2008; Over and Carpenter, 2009).

Social signals not only dictate when and who to mimic, but also carefully control what to mimic for social advantage. Studies revealed that participants show stronger facial mimicry of empathic expressions (e.g., sad) and less facial mimicry of negative expressions (e.g., anger) to ingroup members, compared to outgroup members (Bourgeois and Hess, 2008). This is inconsistent with the simulation theory which predicts that mimicry should not be sensitive to non-imitable features like social membership, but is compatible with the STORM theory. As the authors explain, strong mimicry of empathic facial expressions may signal prosocial intent and thus help to enhance one's affiliation with ingroup members, while reduced mimicry of anger would prevent a spiral of increased aggression and thus aids social harmony. This strategic control of mimicry of different emotions is best explained by the STORM model.

More direct evidence of the STORM theory comes from studies suggesting that the control of mimicry by social signals is strategically driven by the consequence of mimicry. In most cases, mimicry produces positive social consequences 
which help one enhance interpersonal relationship and facilitate social-communication (Chartrand and Bargh, 1999). However in special situations, mimicry may jeopardize the mimicker's social welfare. One example is that mimicry of dominant behaviors to high social status targets makes the mimicker less liking and affiliation (Tiedens and Fragale, 2003). Mastrop et al. (in preparation) revealed that participants decreased mimicry when high social status targets displayed dominant behaviors and increased mimicry when high social status targets displayed status-unrelated behaviors. Karremans and Verwijmeren (2008) showed similar consequence-driven mimicry in another situation where participants who were (or not) involved in a relationship interact with attractive opposite-sex targets. Previous study found that people generally increase mimicry toward attractive opposite-sex targets (van Leeuwen et al., 2009). Here the researchers further proposed that when a man is already in a romantic relationship, increased mimicry toward attractive women other than his romance partner could potentially undermine the current relationship. As they predicted, the results suggest that romantically involved participants displayed decreased mimicry toward opposite-sex targets compared to romantically not-involved participants. This finding suggests that the control of mimicry is sophisticatedly driven by the consequence of mimicry and participants in romance decreased mimicry to shield romantic relationship with their current partner.

Finally, some evidence suggests that the subtly control of mimicry during social interaction can be goal-directed. People implement more mimicry when they have a goal to affiliate with others. For example, in a study by Lakin and Chartrand (2003), participants were either given or not given an affiliation instruction before interacting with a stranger. They found that those participants who were instructed to get along well with the stranger mimicked the stranger to a greater extent than those participants who received no affiliation goal. Similar results were found when participants were implicitly primed by an affiliation goal, such as exposure to a prosocial attitude or interdependent self-construal (Lakin and Chartrand, 2003; van Baaren et al., 2003b; Leighton et al., 2010; Obhi et al., 2011). In another study by Cheng and Chartrand (2003), researchers found that participants who were high self-monitors (i.e., those who are generally strongly motivated to control their own behaviors to leave a good impression to others) displayed more mimicry during social interactions than participants with low self-monitors. These two studies suggest that people tend to display more mimicry when they have certain affiliation goals, whether it is to follow affiliation instructions or to leave a good impression to others. Again, these findings strongly support the STORM account of mimicry that people use mimicry as a behavioral strategy to affiliate with others.

\section{NEW EVIDENCE OF THE STORM THEORY FROM A SOCIAL SRC MIMICRY PARADIGM}

The above-mentioned studies in social psychology primarily adopted a naturalistic paradigm to investigate mimicry in social contexts where participants naturally interact with a confederate whose behaviors or traits were manipulated (Chartrand and van Baaren, 2009). However, these paradigms are limited in their ability to measure the time course of mimicry and to precisely control the social environment. Here we provide new evidence of the STORM in mimicry from recent studies using a novel social stimulus-response compatibility (SRC) paradigm. In this paradigm, participants are required to learn social information about a person by vignettes or videos at first ("social cues stage") and then play a simple SRC task with that person ("social interaction stage") (Figure 1). In each trial of the SRC task, participants observe a simple hand/finger movement by that person and concurrently perform a congruent or incongruent hand/finger movement. As observing an action automatically triggers the tendency to execute that action, previous research found faster responses to congruent than incongruent actions in SRC tasks and took this congruency effect (CE) as a measure of mimicry (see the methodological review by Heyes, 2011a). Thus, researchers can manipulate different social cues about an actor (e.g., animacy, eye gaze, niceness, social status) and test how much participants mimic the actor using the SRC task.

First, the "human bias" feature of mimicry was re-examined by using this social SRC paradigm (Longo and Bertenthal, 2009; Liepelt and Brass, 2010). Previous evidence suggests that mimicry is stronger when the observed action is performed by humans than by non-human agents (see a review by Press, 2011). The underlying mechanism of this effect remains controversial (Press et al., 2006; Stanley et al., 2007, 2010). The domain-general account attributes this animacy effect to the substantial perceptual differences (e.g., kinematics and surface form) between human and non-human action stimuli. It suggests that because people have more sensorimotor experiences with human stimuli than non-human stimuli in the development, action stimuli with human perceptual features elicit stronger motor response than stimuli with non-human perceptual features (Press, 2011). In contrast, the STORM account suggests that the animacy effect primarily comes from the social nature of mimicry, because there would be no need to use mimicry to affiliate with non-human agents. Liepelt and Brass (2010) tested these two accounts by manipulating participants' belief about animacy while keeping the perceptual features constant. They asked participants to complete a finger tapping task (Figure 1, version 2) where identical animation displayed finger movements of a hand in a leather glove. Before running the experiment, half participants were presented with a human hand wearing the leather glove and the other half were presented with a wooden analog hand wearing the leather glove. The results revealed stronger mimicry when participants believed that they interacted with a human hand than when they believed to interact with a wooden hand. As participants with different animacy belief kept equivalent highly loaded attention on stimuli (Leighton et al., 2010) and had the identical perceptual inputs, this finding favors the STORM account suggesting that the animacy effect of mimicry are not from low-level domain-general processes, but from high-level socially specific processes.

Second, we investigated whether the eye gaze of the interaction partner influences mimicry. The STORM theory of mimicry claims that mimicry is driven by its positive social consequences, that is, mimicry facilitates social interaction and enhances liking and affiliation. However, the prerequisite of this affiliative consequence is that the copying behaviors have to be somehow detected by the interaction partner. As eye gaze is a critical social cue that 


\section{Social interaction stage (SRC tasks)}

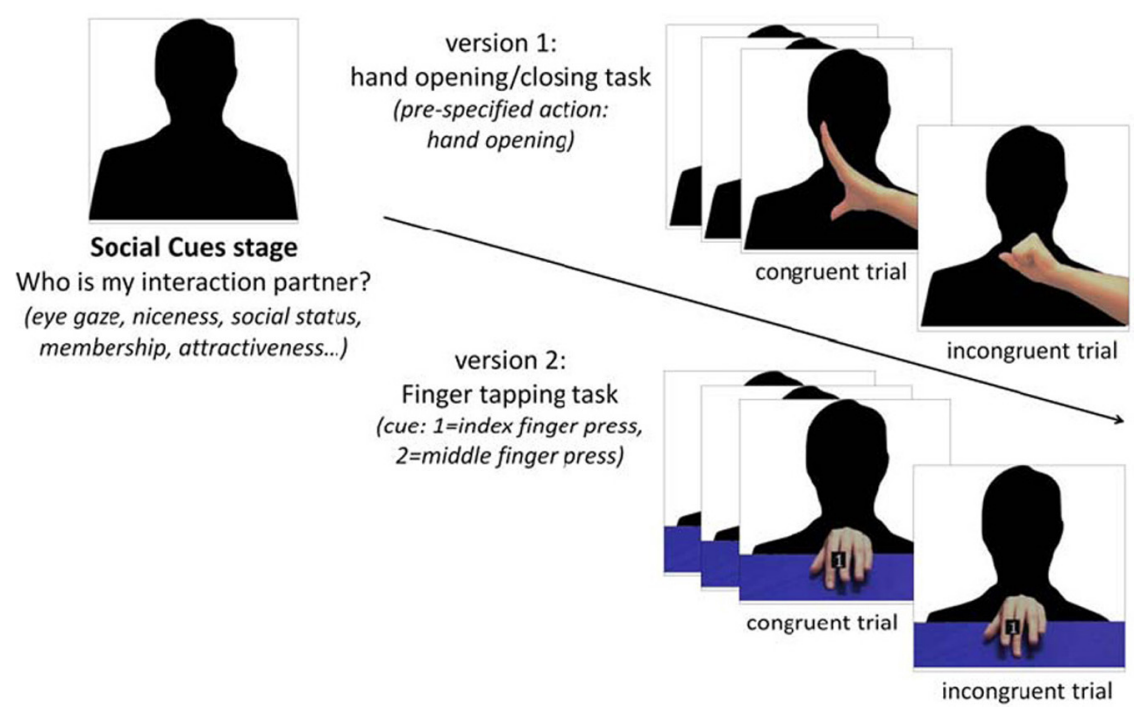

FIGURE 1 | A social SRC paradigm. In this paradigm, participants have to learn about a person (via vignettes or videos) at first ("social cues stage") and then play a SRC task with that person in videos ("social interaction stage"). Two versions of SRC tasks are available: the hand opening/closing task (Heyes et al., 2005) and the finger tapping task (Brass et al., 2000). In hand opening/closing task ("version 1"), participants have to always execute a pre-specified action (e.g., hand open) when the person's hand in the video began to move.
The person's hand could be either open (congruent trials) or close (incongruent trials) but participants always respond by opening their hand. In the finger tapping task ("version 2"), participants have to press a button by using either index or middle finger in response to a number cue (1, index finger; 2 , middle finger) while observing a congruent or incongruent finger movement in the background. Both tasks measure mimicry by calculating the response difference between congruent and incongruent trials.

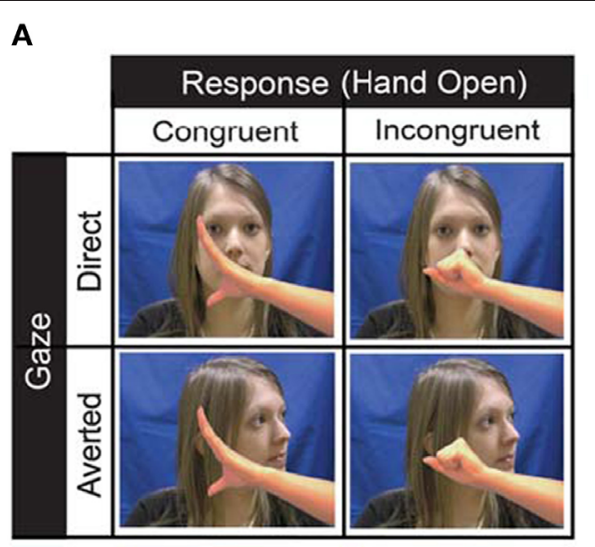

FIGURE 2 | Experimental design (A) and behavioral results (B) of the control of mimicry by eye gaze (Wang et al., 2011a). (A) Participant were shown a series of video clips where an actress did a head movement (direct or averted gaze) and a hand movement. Only the last frame of each video is illustrated here. Participants were required to make the same pre-specified response (e.g., Hand Open) in every stimulus trial in a block, as quickly as possible after the actress' hand in video clips began to move, which could be

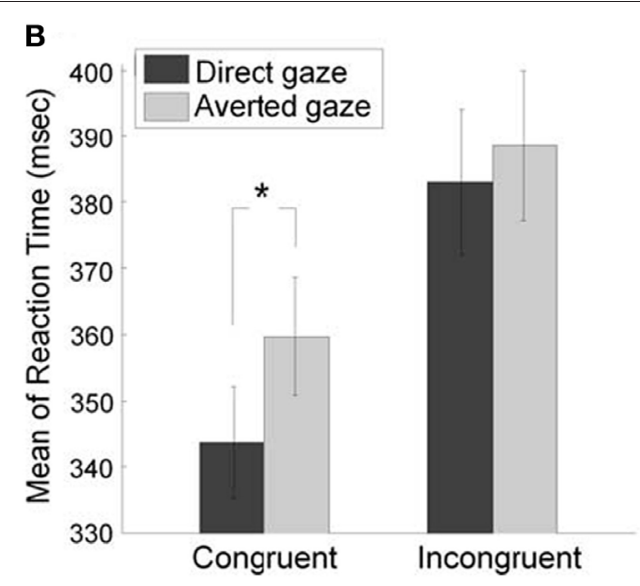

either a hand opening (congruent trials) or hand closing (incongruent trials). As such, each trial fell into a $2 \times 2$ factorial design for direct or averted gaze, congruent or incongruent trial. (B) Mean reaction time on each type of trials. Statistics show that there is a significant difference in the congruency effect between direct and averted gaze conditions, and this difference results from the facilitated congruent trials preceded by direct gaze (the asterisk). conveys the social knowledge of partner's visibility on mimicry behavior, we examined whether mimicry is subtly controlled by partner's gaze direction (Wang et al., 2011a). We adopted our novel social SRC paradigm where participants opened (or closed) their own hand in response to a hand-opening or hand-closing stimuli by an actress (version 1 in Figure 1; Heyes et al., 2005). Critically, before the actress moved her hand, she naturally performed a head movement which resulted in direct gaze or averted gaze (Figure 2A). The results demonstrate that eye gaze rapidly and specifically modulates the mimicry of the hand actions. 
In particular, direct gaze facilitates mimicry of hand action by $13 \mathrm{~ms}$, compared to averted gaze (Figure 2B). These data show that the control of mimicry is fast and selective and that mimicry can be affected by social engagement cues. Thus, we go beyond previous studies showing slower modulation of mimicry by the character of the actor (Chartrand and van Baaren, 2009).

More recently, we have investigated which aspect of eye gaze contributes to this enhancement effect (Wang and Hamilton, in preparation A). As the domain-general theory claims that all modulations of mimicry are through general processes (Heyes, 2012a), we aimed to test whether the control of mimicry by eye gaze is mediated by any attentional processes. Using a similar social SRC paradigm as Wang et al. (2011a), we showed participants a sequence of two gaze events followed by a hand action in the SRC task. In these sequences, the actress' hand was beside (not in front of) her face so that face and hand are spatially separate. The three possible gaze events were: "direct gaze," "averted gaze" and "gaze to the acting hand." Each trial contained two of these three gazes in sequence (e.g., direct-gaze followed by averted-gaze; or averted-gaze followed by hand-gaze), giving a $3 \times 3$ factorial design. One critical trial type involves a joint-attention sequence where the actress provides a direct gaze first and then gazes to the hand. If the enhancement of mimicry is due to joint attention or spatial attention, then mimicry should be strongest in this condition. We contrast this with the three trials where direct gaze was the second event in the sequence (following either direct, averted or hand gaze) and the direct gaze remained during the hand movement cue. If eye contact during action is required for enhancement of mimicry, then the strongest mimicry should be seen in these trials. The results revealed that only the direct-gazeduring-action trials showed mimicry enhancement. This finding rules out explanations of gaze-mimicry interaction in terms of spatial attention and joint attention, and suggests that the social cue of eye contact itself drives mimicry. The eye contact cue could act as an ostensive signal which enhances imitation (Senju and Csibra, 2008; Southgate et al., 2009) or could lead to an "audience effect" where participants are aware they are being watched (Bateson et al., 2006). Further studies will be needed to distinguish these possibilities. Together, these studies of the influence of eye contact on mimicry demonstrate that only direct gaze during action engages a rapid and specific mechanism which enhances the mimicry of hand actions. These findings suggest that the control of mimicry is a social mechanism, not a domain general one, and are compatible with the STORM theory.

Finally, in order to examine whether the effects of social cues on mimicry serve any specific social purposes, we investigated the joint effect of social status and niceness on mimicry (Wang and Hamilton, in preparation B). Participants played the social SRC task (Figure 1, version 2) with four actresses: a nice actress with high status, a nice actress with low status, a nasty actress with high status and a nasty actress with low status. Past studies suggest that high social status (Cheng and Chartrand, 2003; Mastrop et al., in preparation) and nice personality (Likowski et al., 2008; Stel et al., 2010) individually enhance mimicry. If mimicry has no social purposes but only acts as a passive learned response to social stimuli (i.e., "the domain-general theory"), the joint effect should be the summation of individual effect and participants would show greatest mimicry to nice actresses with high status. However, if mimicry acts as an active strategy for social affiliation, participants should show greatest mimicry to those they need to affiliate with but technically challenging to affiliate with, such as the nasty actress with high status. Our results support the latter prediction and found that participants showed greatest mimicry to the nasty actress with high status rather than the nice actress with high status. Again, we suggest that these findings support the STORM theory rather than a simple, stimulus-driven mechanism.

Before finishing this section, we would like to emphasize that our novel social SRC paradigm provides a promising approach for future investigation of the subtlety of mimicry in social contexts. First, some studies have already examined the validity of the SRC paradigm as a measure of mimicry (Heyes, 2011a). It has been suggested that the CE in the SRC paradigms is closely related to the "chameleon effect" in the naturalistic paradigms, and these two paradigms share similar modulative effects by social signals (Lakin and Chartrand, 2003; Leighton et al., 2010; Cook and Bird, 2011a,b; Heyes, 2011a). Second, the novel social SRC paradigm has some advantages over the classic naturalistic paradigm. Social signals are more carefully controlled in the social SRC paradigm. Researchers can accurately manipulate the type, onset and duration of a social signal and measure corresponding mimicry with multiple trials per person in a within-subject design (note that most mimicry studies in naturalistic paradigms are between-subject design). Meanwhile, the social SRC paradigm allows us to investigate the control of mimicry by rapid social cues such as eye gaze (Wang et al., 2011a). Comparing with the naturalistic paradigm which examines modulations of mimicry over a couple of minutes (Chartrand and van Baaren, 2009), the social SRC paradigm optimizes the measurement of control of mimicry into a second-by-second timescale, which is ideal for further application of neuroimaging techniques (Wang et al., 2011b). Finally, the social SRC paradigm can provide us important hints about the underlying mechanisms of the control of mimicry by social signals. As mimicry is measured by the response differences between congruent and incongruent trials, we can roughly infer whether the social signal impacts mimicry process per se (i.e., congruent trials) or the process of inhibition of mimicry (i.e., incongruent trials). For example, in our gaze-mimicry study (Wang et al., 2011a), we found that direct gaze enhances mimicry mainly through the congruent trials rather than incongruent trials (Figure 2B), which suggests that eye gaze directly influences the mimicry process per se, but not the process of the inhibition of mimicry.

In summary, substantial evidence from social psychology and SRC paradigms supports the STORM theory of mimicry, which claims that people strategically control mimicry for their social advantage. Social signals subtly and sophisticatedly guide when and who to mimic and make mimicry behavior more efficient and adaptive. However, what is the neural mechanism underlying this STORM of mimicry?

\section{HOW DO WE MIMIC?}

Without question, it is challenging to directly investigate the neural mechanism of mimicry during social interaction because of its complexity and dynamics. However, understanding why 
we mimic can also contribute to our understanding of how we mimic, and of the brain mechanisms which support flexible and socially meaningful mimicry behavior. We outlined three models above-a STORM model in which mimicry is controlled for social advantage; a simulation model in which mimicry aids interactive alignment, and a domain general model in which mimicry is controlled by general mechanisms. We suggest that these three models can be mapped on to the debate about the relationship between mirror systems and mentalising systems in the human brain. The MNS are located in the inferior frontal gyrus (IFG), inferior parietal cortex (IPL) and superior/middle temporal sulcus/gyrus (STS/MTG), and are engaged by both observation and execution of action (Iacoboni et al., 1999; Rizzolatti and Craighero, 2004). They have been strongly linked to the implementation of mimicry and other visual-motor responses (Iacoboni et al., 1999; Catmur et al., 2008, 2009; Bien et al., 2009; Heyes, 2011a). Mentalising systems are found in temporo-parietal junction (TPJ) and MPFC and are engaged when participants must judge other people's mental states or other social features (van Overwalle and Baetens, 2009). The relationship between these two brain networks and their relative roles in social cognition is a matter of much debate (Brass et al., 2007; Csibra, 2007; Uddin et al., 2007; Hickok, 2009).

We suggest that the interactions between these two brain networks during mimicry are different for each model (Figure 3). If mirror systems respond to and mimic an observed action, and this information feeds up to mentalising systems, this represents a simulation process (Figure 3, arrow A). The action representation in the mirror systems aids interactive alignment and thus enhances mentalising. In contrast, if social judgements from mentalising systems are used to control the implementation of mimicry in the mirror system, this represents a top-down control process, i.e., STORM (Figure 3, arrow B). Social evaluation from mentalising system acts as a controller of mimicry. If mirror systems and mentalising system are independent during mimicry, this favors the domain-general model suggesting that mimicry is not controlled by any specific social processes.

All three models encompass current theories suggesting that mimicry arises from associative learning (the ASL model, Heyes, 2001), and focus instead on how basic mimicry processes relate to other components of social cognition (Figure 3). Distinguishing these models will help to determine why people mimic and will define the neural mechanisms underlying mimicry. Here, we will review previous evidence of the brain systems for the inhibition of mimicry and introduce our recent neuroimaging data on the brain systems for the social control of mimicry. We suggest that the evidence favors the STORM model.

\section{NEURAL MECHANISMS OF THE INHIBITION OF MIMICRY}

To some extent, the implementation of mimicry by the MNS makes mimicry act as a prepotent response tendency-observing an action automatically triggers the tendency to execute that action. Since mimicry is not adaptive in every situation, the question that arises is how such a tendency to mimic is prevented from leading to over-mimicry symptoms such as echolalia and echopraxia (i.e., excessive repetition of other's speech or observed actions) or inappropriate mimicry such as copying

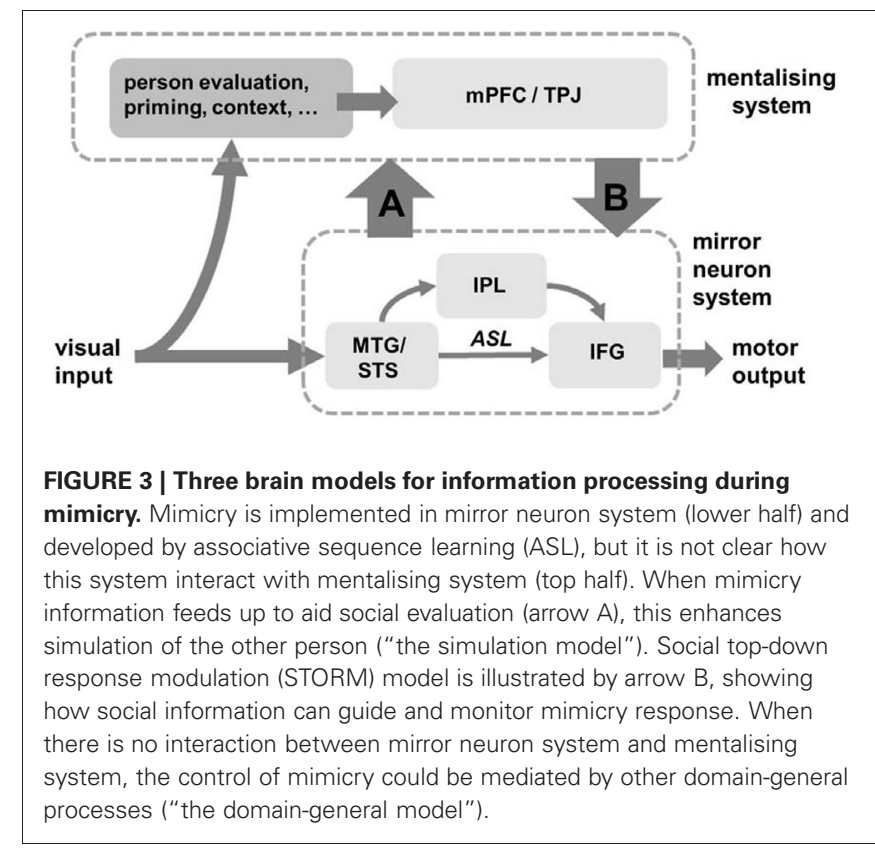

dominant behaviors from high status people. Moreover, a simple direct-mapping mechanism provided by the MNS cannot fully explain the complexity and dynamic of mimicry in social interactions (Southgate and Hamilton, 2008). As mimicry is subtly and sophisticatedly controlled by social signals, there must be some controlling systems supervising the MNS according to social contexts.

Early clinical observation suggests that the inhibition of inappropriate response tendencies is a function of the prefrontal lobes. Patients with prefrontal lesions have difficulties in tasks involving inhibition of prepotent responses (such as Stroop task and the go/no-go paradigm) and sometimes display over-mimicry symptoms such as echolalia and echopraxia (Luria, 1980; Lhermitte et al., 1986; Vendrell et al., 1995; De Renzi et al., 1996). Later neuroimaging studies using the classic SRC paradigm support this observation and further suggest $\mathrm{MPFC}$ and TPJ are two key brain regions for the inhibition of mimicry (Brass et al., 2001, 2005). Stronger activations in mPFC and TPJ were observed when participants have to inhibit their natural tendency to mimic in incongruent trials. Interestingly, these two regions are both anatomically and functionally different from the systems responsible for the Stroop task (e.g., dorsolateral PFC, ACC) (Brass et al., $2003,2005)$. This shows that control of imitation is distinct from other simple forms of cognitive control, and thus argues against a domain-general theory of the control of mimicry.

Recent studies suggest that the inhibition of mimicry is closely associated with mentalising processes (Brass et al., 2009). As $\mathrm{mPFC}$ and TPJ are two brain regions that engage in both mentalising tasks (Frith and Frith, 1999) and inhibition of mimicry tasks (Brass et al., 2001, 2005), Brass and his colleagues (2009) proposed that these two processes are linked. They did several behavioral studies to test this idea. In the first study, Spengler et al. (2010c) implemented the SRC and Theory of Mind tasks in both healthy participants and neuropsychological patients with 
prefrontal lesions or TPJ lesions, to examine whether there is a functional association between the inhibition of mimicry and mentalising abilities. The results showed a highly significant correlation between mimicry control and the abilities to mental attribution in both health participants and patient with lesions. In the second study, Spengler et al. (2010a) implemented the same experiment design on individuals with autism, who are known to have difficulties with mentalising. Similar to those patients with prefrontal or TPJ lesions, the results suggest that mentalising abilities in autism are positively correlated with their inhibition of mimicry: the worse individuals with autism performed in the mentalising tasks, the less they were able to inhibit their automatic mimicry. A key question arises here: what is the cognitive overlap between mentalising and inhibition of mimicry? Brass et al. (2009) suggest that both require good self-other distinction. In the third study, Spengler et al. (2010b) directly manipulated the processes for self-other distinction when healthy participants were performing the SRC task. In half of the blocks, participants were asked to do the task with two mirrors placed on each side of the monitor, so that participants could see their face and upper part of the body reflected in the mirror ("selffocus" condition). In the other half blocks, the mirrors were turned around with the non-reflective side facing the subjects ("no self-focus" condition). Results showed that comparing with no self-focus conditions, participants showed stronger inhibition of mimicry during self-focus conditions, which suggests that increasing self-other distinction leads to enhanced inhibition of mimicry. Interestingly, a recent study by Santiesteban et al. (2012) supports this close relationship in the other direction. They trained participants to inhibit the tendency to mimic and then measured their performance in tasks requiring selfother distinction. They found that, compared with no-training groups, participants with inhibition training showed improved performance on a visual perspective-taking task, which suggests that enhanced inhibition of mimicry leads to enhanced self-other distinction process. To sum up, these findings consistently suggest that the inhibition of mimicry and mentalising processes all share cognitive components such as the self-other distinction in $\mathrm{mPFC}$ and TPJ. These components are specifically social, not domain-general.

\section{SOCIAL CONTROL OF MIMICRY}

As outlined above, the inhibition of mimicry is linked to some types of social information processing such as the self-other distinction. However, this does not explain the information processing behind the decision of when to mimic and when to inhibit mimicry. Behavioral studies show that mimicry responses change dependent on the social context, but it is not yet clear how this could be implemented. Again, recent data suggests that $\mathrm{mPFC}$ responds to social cues such as eye gaze and social status (Kampe et al., 2003; Singer et al., 2004; Zink et al., 2008) and has a key role in monitoring other social processes (Teufel et al., 2010). mPFC is also an important region in monitoring of task responses associated with social rewards or punishment (Amodio and Frith, 2006). Thus, we suggest that mPFC could be a good candidate for implementing STORM in many social contexts, though other components of the social brain are also likely to contribute.
We tested this idea by examining the neural mechanism of the eye contact effect on mimicry (Wang et al., 2011b). Participants performed a social SRC task with eye gaze priming as Wang et al. (2011a) (see Figure 2) during fMRI scanning. The fMRI results showed that performing the SRC task activated the MNS while observation of direct gaze and inhibition of mimicry both engaged mPFC. These findings were consistent with previous studies (Brass et al., 2001, 2005; Senju and Johnson, 2009). Critically, three brain regions showed an interaction between the eye gaze present on a trial and whether mimicry was inhibited or enhanced. These were the mirror system regions STS and IFG, together with $\mathrm{mPFC}$ (Figure 4A). This result is compatible with our hypothesis that mPFC is controlling mimicry implemented by STS and IFG.

We then used dynamic causal modeling (DCM) to investigate the information processing between these regions (Figure $4 \mathbf{B}$ ). In the optimal model, three features stand out. First, there was strong intrinsic connectivity strength from mPFC to IFG and from mPFC to STS (solid white arrows in Figure 4B), which suggests that mPFC constantly exerts top-down control on the MNS. Second, when participants performed the SRC task (compared to a baseline task), the connectivity strength from STS to IFG increased, suggesting that these regions implement the visuomotor mapping for the task. Finally, the interaction of direct gaze and mimicry enhances the connection strength from mPFC to STS, which suggests that MPFC is the originator of the gazemimicry interaction and that it modulates sensory input (i.e., STS) to the MNS. This study is the first to investigate the underlying neural mechanism of the control of mimicry by social signals, and demonstrated that mPFC subtly controls mimicry according to gaze directions by modulating the sensory input of the MNS. It demonstrated how different components of the social brain work together to control mimicry according to the gaze direction. It provides strong support for the STORM model of mimicry that social judgments from mentalising systems are used to top-down control mimicry. In the future, it will be interesting to test whether other social cues modulate mimicry through the mediation of mPFC and whether other brain regions for social evaluation/monitoring (e.g., ventral striatum, basolateral amygdala) are also involved in the STORM of mimicry (Singer et al., 2004; Zink et al., 2008).

\section{IMPLICATIONS AND FUTURE DIRECTIONS}

The STORM model of mimicry we discussed in this paper has several important implications for our understanding of mimicry and human social behavior. First, the claim that mimicry is socially top-down modulated means that even this rapid, unconscious, learning-dependent behavior is subtly controlled by social goals. This control seems to include the somewhat Machiavellian goal of increasing one's social standing and welfare. The STORM of mimicry could be built on top of non-specific associative learning mechanism (Heyes, 2001) but goes beyond it with dedicated social mechanisms that use mimicry as a social strategy. The evidence we reviewed above hint at the remarkable sophistication of this mechanism-it can implement both inhibition and enhancement of mimicry (Wang et al., 2011b), and it evaluates the social meaning of each event rather than simply responding 


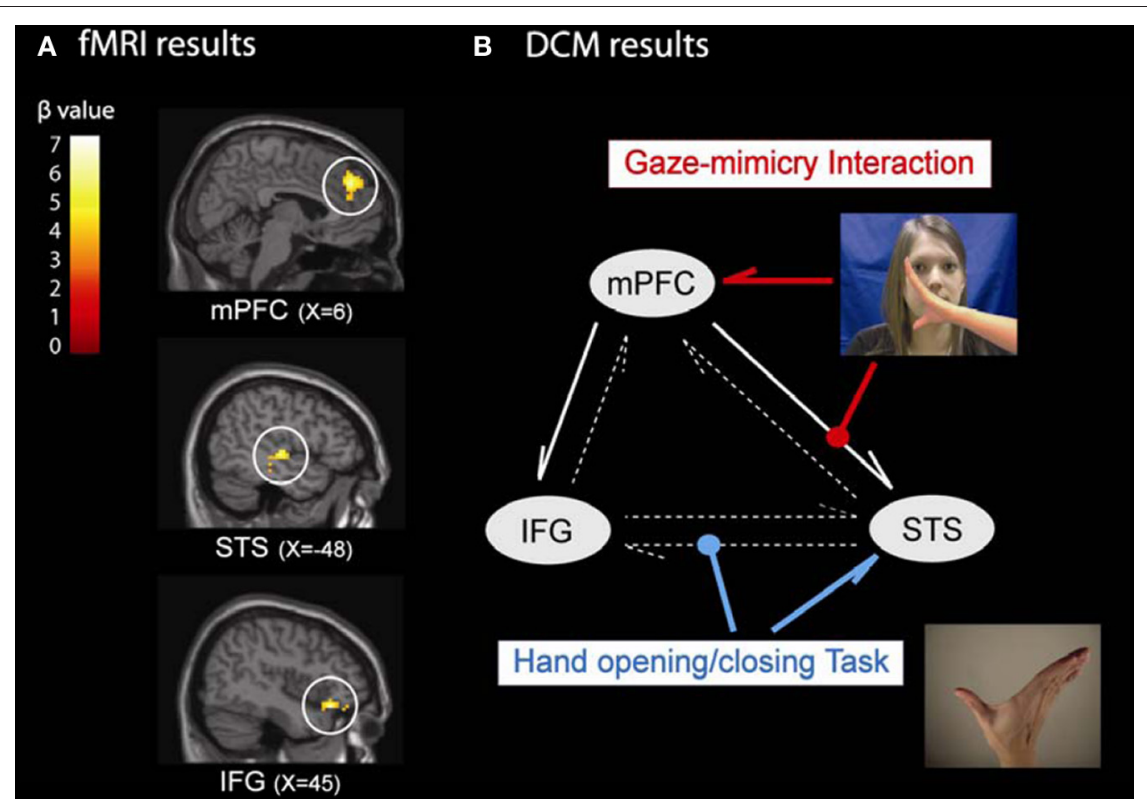

FIGURE 4 | The neural mechanism of the eye contact effect on mimicry. (A) the $f M R I$ results showed three brain regions specifically for the gaze-mimicry interaction: mPFC, STS, and IFG; and $(\mathbf{B})$ the DCM further suggested a best model where the mimicry increases the connectivity strength from STS to IFG during the SRC task (blue line) and the direct gaze controls mimicry by modulating the connection strength from mPFC to STS (red line). to positive stimulus features (Wang and Hamilton, in preparation B). Further work will be needed to define the limits of mimicry control and to determine how different types of social information and social goals contribute to this process. In particular, the relationship between the modulation of mimicry by semantic priming (Lakin and Chartrand, 2003; Leighton et al., 2010) and the control of mimicry by social cues remains unclear.

It is also important to consider what might happen when the control of mimicry breaks down. As mentioned above, both echopraxia/echolalia and utilisation behavior are seen in patients with prefrontal lobe damage, suggesting poor control of prepotent responses in this group (De Renzi et al., 1996). Deficits in imitation and mimicry have also been reported in participants with autistic spectrum disorder (Williams et al., 2004), and some have linked this to the functioning of the MNS (Williams et al., 2001; Oberman et al., 2005; Oberman and Ramachandran, 2007). However, increasing evidence points to typical mirror neuron responses in participants with autism (Dinstein et al., 2010; Marsh and Hamilton, 2011), meaning that a "broken mirror system" cannot be the origin of poor mimicry in autism (Southgate and Hamilton, 2008). As STORM of mimicry is important for functional mimicry and one's social competency, here we suggest that the control of mimicry might be abnormal in autistic individuals. It is now known that the $\mathrm{MPFC}$, the key brain region for the control of mimicry, is abnormal in autism (Kennedy et al., 2006; Gilbert et al., 2009). Recent data from (Cook and Bird, $2011 \mathrm{a}, \mathrm{b})$ directly suggests that abnormality of mPFC in autistic populations or immaturity of mPFC in adolescents lead to poor control of mimicry. Thus, it is plausible to suggest that poor social top-down control of mimicry is responsible for abnormal mimicry behavior in autism (Hamilton, 2008). Further research will be needed to test this possibility.

\section{CONCLUSION}

This paper has evaluated theories of why people mimic and the brain systems that implement mimicry. We suggest that current data favor a STORM model of mimicry, in which mimicry is carefully controlled to maximize one's social advantage. Recent fMRI data implicate mPFC in this control process. Future studies should examine how different types of social information are used in the control of mimicry and whether the control of mimicry is abnormal in autism.

\section{REFERENCES}

Amodio, D. M., and Frith, C. D. (2006). Meeting of minds: the medial frontal cortex and social cognition. Nat. Rev. Neurosci. 7, 268-277.

Bateson, M., Nettle, D., and Roberts, G. (2006). Cues of being watched enhance cooperation in a real-world setting. Biol. Lett. 2, 412-414.
Bien, N., Roebroeck, A., Goebel, R., and Sack, A. T. (2009). The brain's intention to imitate: the neurobiology of intentional versus automatic imitation. Cereb. Cortex 19, 2338-2351.

Bourgeois, P., and Hess, U. (2008). The impact of social context on mimicry. J. Nonverbal Behav. 77, 343-352.
Brass, M., Bekkering, H., Wohlschlager, A., and Prinz, W. (2000). Compatibility between observed and executed finger movements: comparing symbolic, spatial, and imitative cues. Brain Cogn. 44, 124-143.

Brass, M., Derrfuss, J., and von Cramon, D. Y. (2005). The inhibition of imitative and overlearned responses: a functional double dissociation. Neuropsychologia 43, 89-98.

Brass, M., Derrfuss, J., von Cramon, G. M., and von Cramon, D. Y. (2003). Imitative response tendencies in patients with frontal brain lesions. Neuropsychologia 17, 265-271. 
Brass, M., and Heyes, C. (2005). Imitation: is cognitive neuroscience solving the correspondence problem? Trends Cogn. Sci. 9, 489-495.

Brass, M., Ruby, P., and Spengler, S. (2009). Inhibition of imitative behaviour and social cognition. Philos. Trans. R. Soc. Lond. B Biol. Sci. 364, 2359-2367.

Brass, M., Schmitt, R. M., Spengler, S., and Gergely, G. (2007). Investigating action understanding: inferential processes versus action simulation. Curr. Biol. 17, 2117-2121.

Brass, M., Zysset, S., and von Cramon, D. Y. (2001). The inhibition of imitative response tendencies. Neuroimage 14, 1416-1423.

Catmur, C., Gillmeister, H., Bird, G., Liepelt, R., Brass, M., and Heyes, C. M. (2008). Through the looking glass: counter-mirror activation following incompatible sensorimotor learning. Eur. J. Neurosci. 28, 1208-1215.

Catmur, C., Walsh, V., and Heyes, C. M. (2007). Sensorimotor learning configures the human mirror system. Curr. Biol. 17, 1527-1531.

Catmur, C., Walsh, V., and Heyes, C. M. (2009). The role of experience in the development of imitation and the mirror system. Philos. Trans. R. Soc. Lond. B Biol. Sci. 364 , 2369-2380.

Chartrand, T. L., and Bargh, J. A. (1999). The chameleon effect: the perception behavior link and social interaction. J. Pers. Soc. Psychol. 76, 893-910.

Chartrand, T. L., and van Baaren, R. (2009). Human mimicry. Adv. Exp. Soc. Psychol. 41, 219-274.

Cheng, C. M., and Chartrand, T. L. (2003). Self-monitoring without awareness: using mimicry as a nonconscious affiliation strategy. J. Pers. Soc. Psychol. 85, 1170-1179.

Chong, T. T., Cunnington, R., Williams, M. A., and Mattingley, J. B. (2009). The role of selective attention in matching observed and executed actions. Neuropsychologia 47, 786-795.

Cook, J., and Bird, G. (2011a). Social attitudes differently modulate imitation in adolescents and adults. Exp. Brain Res. 211, 601-612.

Cook, J., and Bird, G. (2011b). Atypical social modulation of imitation in autism spectrum conditions. J. Autism Dev. Disord. Advance online publication. doi: 10.1007/s10803011-1341-7

Cook, R., Dickinson, A., and Heyes, C. (2012). Contextual modulation of mirror and counter-mirror sensorimotor associations. J. Exp. Psychol. Gen. Advance online publication. doi: 10.1037/a0027561

Csibra, G. (2007). "Action mirroring and action understanding: an alternative account," in Sensorimotor Foundations of Higher Cognition: Attention and Performance, Vol. XXII, eds P. Haggard, Y. Rossetti, and M. Kawato (Oxford: Oxford University Press), 435-480.

De Renzi, E., Cavalleri, F., and Facchini, S. (1996). Imitation and utilisation behaviour. J. Neurol. Neurosurg. Psychiatry 61, 396-400.

Dinstein, I., Thomas, C., Humphreys, K., Minshew, N., Behrmann, M., and Heeger, D. J. (2010). Normal movement selectivity in autism. Neuron 66, 461-469.

Frith, C. D., and Frith, U. (1999). Interacting minds-biological basis. Science 286, 1692-1695.

Gallese, V. (2007). Before and below 'theory of mind': embodied simulation and the neural correlates of social cognition. Philos. Trans. R. Soc. Lond. B Biol. Sci. 362, 659-669.

Gallese, V. (2009). Mirror neurons, embodied simulation, and the neural basis of social identification. Psychoanal. Dialogues 19, 519-536.

Gilbert, S. J., Meuwese, J. D. I., Towgood, K. J., Frith, C. D., and Burgess, P. W. (2009). Abnormal functional specialization within medial prefrontal cortex in highfunctioning autism: a multi-voxel similarity analysis. Brain 132, 869-878.

Hamilton, A. (2008). Emulation and mimicry for social interaction: a theoretical approach to imitation in autism. Q. J. Exp. Psychol. 61, 101-115.

Hari, R., and Kujala, M. V. (2009). Brain basis of human social interaction: from concepts to brain imaging. Physiol. Rev. 89, 453-479.

Hennenlotter, A., Dresel, C. Castrop, F., Baumann, A. O. C., Wohlschlager, A. M., and Haslinger, B. (2009). The link between facial feedback and neural activity within central circuitries of emotion: new insights from botulinum toxininduced denervation of frown muscles. Cereb. Cortex 19, 537-542.

Heyes, C. M. (2001). Causes and consequences of imitation. Trends $\operatorname{Cog} n$. Sci. 5, 253-261.

Heyes, C. M. (2009). Evolution, development and intentional control of imitation. Philos. Trans. R. Soc. Lond. B Biol. Sci. 364, 2293-2298.

Heyes, C. M. (2011a). Automatic imitation. Psychol. Bull. 137, 463-483.

Heyes, C. M. (2011b). What's social about social learning? J. Comp.
Psychol. Advance online publication. doi:10.1037/a0025180.

Heyes, C. M. (2012a). "What can imitation do for cooperation?" in Signalling, Commitment and Emotion, eds B. Calcott, R. Joyce, and K. Sterelny (Cambridge, MA: MIT Press)

Heyes, C. M. (2012b). "Imitation associative and context-dependent," in Tutorials in Action Science, eds W. Prinz, M. Beisert, and A. Herwig (Cambridge, MA: MIT Press).

Heyes, C. M., Bird, G., Johnson, H., and Haggard, P. (2005). Experience modulates automatic imitation. Cogn. Brain Res. 22, 233-240.

Hickok, G. (2009). Eight problems for the mirror neuron theory of action understanding in monkeys and humans. J. Cogn. Neurosci. 21, 1229-1243.

Iacoboni, M., Woods, R. P., Brass, M., Bekkering, H., Mazziotta, J. C., and Rizzolatti, G. (1999). Cortical mechanisms of human imitation. Science 286, 2526-2528.

Kampe, K. K., Frith, C. D., and Frith, U. (2003). "Hey John": signals conveying communicative intention toward the self activate brain regions associated with "mentalizing," regardless of modality. J. Neurosci. 23, 5258-5263.

Karremans, J. C., and Verwijmeren, T. (2008). Mimicking attractive opposite-sex others: the role of romantic relationship status. Pers. Soc. Psychol. Bull. 34, 939-950.

Kennedy, D. P., Redcay, E., and Courchesne, E. (2006). Failing to deactivate: resting functional abnormalities in autism. Proc. Natl. Acad. Sci. U.S.A. 103, 8275-8280.

Lakin, J. L., and Chartrand, T. L. (2003). Using nonconscious behavioral mimicry to create affiliation and rapport. Psychol. Sci. 14, 334-339.

Lakin, J. L., Chartrand, T. L., and Arkin, R. M. (2008). I am too just like you: the effects of ostracism on nonconscious mimicry. Psychol. Sci. 19, 816-822.

Lakin, J. L., Jefferis, V. E., Cheng, C. M., and Chartrand, T. L. (2003). The chameleon effect as social glue: evidence for the evolutionary significance of nonconscious mimicry. J. Nonverbal Behav. 27, 145-162.

Leighton, J., Bird, G., Orsini, C., and Heyes, C. (2010). Social attitudes modulate automatic imitation. $J$ Exp. Soc. Psychol. 46, 905-910.

Lhermitte, F., Pillon, B., and Serdaru, M. (1986). Human autonomy and the frontal lobes. Part I: imitation and utilization behavior: neuropsychological study of 75 patients. Ann. Neurol. 19, 326-334.

Liepelt, R., and Brass, M. (2010). Topdown modulation of motor priming by belief about animacy. Exp. Psychol. 57, 221-227.

Likowski, K. U., Mühlberger, A., Seibt, B., Pauli, P., and Weyers, P. (2008). Modulation of facial mimicry by attitudes. J. Exp. Soc. Psychol. 44, 1065-1072.

Longo, M. R., and Bertenthal, B. I. (2009). Attention modulates the specificity of automatic imitation to human actors. Exp. Brain Res. 192, 739-744.

Luria, A. R. (1980). Higher Cortical Functions in Man, 2nd edn. New York, NY: Basic books.

Maddux, W. W., Mullen, E., and Galinsky, A. D. (2008). Chameleons bake bigger pies and take bigger pieces: strategic behavioral mimicry facilitates negotiation outcomes. J. Exp. Soc. Psychol. 44, 461-468.

Marsh, L. E., and Hamilton, A. F. (2011). Dissociation of mirroring and mentalising systems in autism. Neuroimage 56, 1511-1519.

Niedenthal, P. M., Mermillod, M., Maringer, M., and Hess, U. (2010). The Simulation of smiles (SIMS) model: embodied simulation and the meaning of facial expression. Behav. Brain Sci. 33, 417-480.

Oberman, L. M., Hubbard, E. M., McCleery, J. P., Altschuler, E. L., Ramachandran, V. S., and Pineda, J. A. (2005). EEG evidence for mirror neuron dysfunction in autism spectrum disorders. Cogn. Brain Res. 24, 190-198.

Oberman, L. M., and Ramachandran, V. S. (2007). The simulating social mind: the role of the mirror neuron system and simulation in the social and commu- nicative deficits of autism spectrum disorder. Psychol. Bull. 133, 310-327.

Obhi, S. S., Hogeveen, J., and PascualLeone, A. (2011). Resonating with others: the effects of self-construal type on motor cortical output. J. Neurosci. 41, 14531-14535.

Over, H., and Carpenter, M. (2009). Priming third-party ostracism increases affiliative imitation in children. Dev. Sci. 12, F1-F8.

Pickering, M. J., and Garrod, S. (2004). Toward a mechanistic psychology of dialogue. Behav. Brain Sci. 27, 169-225.

Press, C. (2011). Action observation and robotic agents: learning and anthropomorphism. Neurosci. Biobehav. Rev. 35, 1410-1418.

Press, C., Gillmeister, H., and Heyes, C. M. (2006). Bottom-up, not top-down, modulation of imitation 
by human and robotic models. Eur. J. Neurosci. 24, 2415-2419.

Press, C., Heyes, C. M., and Kilner, J. M. (2011). Learning to understand others' actions. Biol. Lett. 7, 457-460.

Rizzolatti, G., and Craighero, L. (2004). The mirror-neuron system. Annu. Rev. Neurosci. 27, 169-192.

Santiesteban, I., White, S., Cook, J., Gilbert, S. J., Heyes, C. M., and Bird, G. (2012). Training social cognition: from imitation to theory of mind. Cognition 122, 228-235.

Senju, A., and Csibra, G. (2008). Gaze following in human infants depends on communicative signals. Curr. Biol. 18, 668-671.

Senju, A., and Johnson, M. H. (2009). The eye contact effect mechanisms and development. Trends Cogn. Sci. 13, 127-134.

Singer, T., Kiebel, S. J., Winston, J. S. Dolan, R. J., and Frith, C. D. (2004). Brain responses to the acquired moral status of faces. Neuron 41, 653-662.

Southgate, V., Chevalier, C., and Csibra, G. (2009). Sensitivity to communicate relevance tells young children what to imitate. Dev. Sci. 12, 1013-1019.

Southgate, V., and Hamilton, A. F. (2008). Unbroken mirrors: challenging a theory of Autism. Trends Cogn. Sci. 12, 225-229.

Spengler, S., Bird, G., and Brass, M. (2010a). Hyper-imitation of actions is related to reduced understanding of others' minds in autism spectrum conditions. Biol. Psychiatry 68, 1148-1155.

Spengler, S., Brass, M., Kühn, S., and Schütz-bosbach, S. (2010b). Minimizing motor mimicry by myself: self-focus enhances online action-control mechanisms during motor contagion. Conscious. Cogn. 19, 98-106.
Spengler, S., von Cramon, D. Y., and Brass, M. (2010c). Resisting motor mimicry: control of imitation involves processes central to social cognition in patients with frontal and temporo-parietal lesion. Soc. Neurosci. 19, 98-106.

Stanley, J., Gowen, E., and Miall, R. C. (2007). Effects of agency on movement interference during observation of amoving dot stimulus. J. Exp. Psychol. Hum. Percept. Perform. 33, 915-926.

Stanley, J., Gowen, E., and Miall, R. C. (2010). How instructions modify perception: an fMRI study investigating brain areas involved in attributing human agency. Neuroimage 52, 389-400.

Stel, M., van Baaren, R. B., Blascovich, J., van Dijk, E., McCall, C., Pollmann, M. M. H., van Leeuwen, M. L., Mastop, J., and Vonk, R. (2010). Effects of a priori liking on the elicitation of mimicry. Exp. Psychol. 57, 412-418.

Stel, M., and van Knippenberg, A. (2008). The role of facial mimicry in the recognition of affect. Psychol. Sci. 19, 984-985.

Teufel, C., Fletcher, P. C., and Davis, G. (2010). Seeing other minds: attributed mental status influence perception. Trends Cogn. Sci. 14, 376-382.

Tiedens, L. Z., and Fragale, A. R. (2003). Power moves: complementarity in dominant and submissive nonverbal behavior. J. Pers. Soc. Psychol. 84, 558-568.

Uddin, L. Q., Iacoboni, M., Lange, C., and Keenan, J. P. (2007). The self and social cognition: the role of cortical midline structures and mirror neurons. Trends Cogn. Sci. 11, 153-157.

van Baaren, R., Holland, R. W., Kawakami, K., and van
Knippenberg, A. (2004). Mimicry and prosocial behavior. Psychol. Sci. 15, 71-74.

van Baaren, R., Holland, R. W. Steenaert, B., and van Knippenberg, A. (2003a). Mimicry for money: behavioral consequences of imitation. J. Exp. Soc. Psychol. 39, 393-398.

van Baaren, R., Janssen, L., Chartrand, T. L., and Dijksterhuis, A. (2009). Where is the love? The social aspects of mimicry. Philos. Trans. R. Soc. Lond. B Biol. Sci. 364 2381-2389.

van Baaren, R., Maddux, W. W. Chartrand, T. L., de Bouter, C., and van Knippenberg, A. (2003b). It takes two to mimic: behavioral consequences of self-construals. J. Pers. Soc. Psychol. 84, 1093-1102.

van Leeuwen, M. L., Veling, H., van Baaren, R. B., and Dijksterhuis, A. (2009). The influence of facial attractiveness on imitation. J. Exp. Soc. Psychol. 45, 1295-1298.

van Overwalle, F., and Baetens, K. (2009). Understanding others' actions and goals by mirror and mentalizing systems: a meta-analysis. Neuroimage 48, 564-584.

Vendrell, P., Junque, C., Pujol, J., Jurado, M. A., Molet, J., and Grafman, J. (1995). The role of prefrontal regions in the stroop task. Neuropsychologia 33, 341-352.

Wang, Y., Newport, R., and Hamilton, A. (2011a). Eye contact enhances mimicry of intransitive hand movements. Biol. Lett. 7, 7-10.

Wang, Y., Ramsey, R., and Hamilton, A. (2011b). The control of mimicry by eye contact is mediated by medial prefrontal cortex. J. Neurosci. 31, 12001-12010.

Williams, J. H., Whiten, A., and Singh, T. (2004). A systematic review of action imitation in autistic spectrum disorder. J. Autism Dev. Disord. 34, 285-299.

Williams, J. H., Whiten, A., Suddendorf, T., and Perrett, D. I. (2001). Imitation, mirror neurons and autism. Neurosci. Biobehav. Rev. 25, 287-295.

Yabar, Y., Johnston, L., Miles, L., and Peace, V. (2006). Implicit behavioral mimicry: investigating the impact of group membership. J. Nonverbal Behav. 30, 97-113.

Zink, C. F., Tong, Y., Chen, Q., Bassett, D. S., Stein, J. L., and MeyerLindenberg, A. (2008). Know your place: neural processing of social hierarchy in humans. Neuron 58, 273-283.

Conflict of Interest Statement: The authors declare that the research was conducted in the absence of any commercial or financial relationships that could be construed as a potential conflict of interest.

Received: 23 January 2012; paper pending published: 27 February 2012; accepted: 16 May 2012; published online: 04 June 2012

Citation: Wang $Y$ and Hamilton AF (2012) Social top-down response modulation (STORM): a model of the control of mimicry in social interaction. Front. Hum. Neurosci. 6:153. doi: 10.3389/ fnhum.2012.00153

Copyright (c) 2012 Wang and Hamilton. This is an open-access article distributed under the terms of the Creative Commons Attribution Non Commercial License, which permits non-commercial use, distribution, and reproduction in other forums, provided the original authors and source are credited. 